\title{
End to End Performance Analysis of Relay Cooperative Communication Based on Parked Cars
}

\author{
Yingying SUN*, Lijun WANG ${ }^{\dagger \ddagger}$, Zhiquan BAI ${ }^{\S}$, Kyung Sup KWAK ${ }^{\Uparrow}$, Xuming YAO* and Tao HAN* \\ ${ }^{*}$ School of Electronic Information and Communications, Huazhong University of Science and Technology, China \\ ${ }^{\dagger}$ School of Electronic Information, Wuhan University, China \\ $\ddagger$ Department of Information Science and Technology, Wenhua College, China \\ $\S$ School of Information Science and Engineering, Shandong University, China \\ ฯ Inha Hanlim Fellow Professor, Department of Information and Communication, Inha university, Korea \\ sunny_hbqq@hust.edu.cn, wanglj22@163.com,zqbai@sdu.edu.cn, kskwak@inha.ac.kr, yaoxuming@hust.edu.cn, \\ hantao@hust.edu.cn
}

\begin{abstract}
Parking lots (PLs) are usually full with cars. If these cars are formed into a self-organizing vehicular network, they can be new kind of road side units (RSUs) in urban area to provide communication data forwarding between mobile terminals nearby and a base station. However cars in PLs can leave at any time, which is neglected in the existing studies. In this study, we investigate relay cooperative communication based on parked cars in PLs. Taking the impact of the car's leaving behavior into consideration, we derive the expressions of outage probability in a two-hop cooperative communication and its link capacity. Finally, the numerical results show that the impact of a car's arriving time is greater than the impact of its parking duration on outage probability.
\end{abstract}

Keywords-Parking Lots, Leaving Behavior, Outage Probability, Cooperative Communication, Vehicular Networks

\section{INTRODUCTION}

Vehicular ad hoc network (VANET) is an important part of the future smart city, which can support services such as data forwarding, content caching and mobile edge computation [1], [2]. Studies on vehicular network in the past mainly concentrated on moving cars. With the number of worldwide vehicles increasing rapidly, and most of cars spend most of the time in a day on parking, there are large numbers of parked cars in urban areas. Regarding these parked vehicles as an infrastructure, we can make use of their own wireless devices and CPUs to improve the communication quality and computation ability in urban vehicular network [3]. Some studies have proposed the idea of introducing parked cars into vehicular networks as static nodes for relay forwarding [4], [5] and content caching [6], [7], [8]. In the intelligent transportation system, moving vehicles on the road always need to share safety message with each other. Studies show

The corresponding author is Tao Han. The authors would like to acknowledge the support of the International Science and Technology Cooperation Program of China (Grant No. 2015DFG12580), the National Natural Science Foundation of China (Grant Nos. 61471180 and 61771291), the Fundamental Research Funds of Shandong University (Grant No. 2016JC010), the Nationa Research Foundation of Korea-Grant funded by the Korean Government (Ministry of Science, ICT and Future Planning)-NRF-2014K1A3A1A20034987), and the EU FP7-PEOPLE-IRSES (Grant No. 610524). This research is supported in part by the China International Joint Research Center of Green Communications and Networking (No. 2015B01008). that there will take at least 100 seconds to broadcast important safety message to all nearby cars, if in areas of low car density [9]. One way to overcome it is to deploy roadside units (RSUs) alongside roads, which brings high installation and maintenance costs. Therefore, some researchers proposed the idea of regarding parked cars as temporary RSUs [10], [11]. Besides, parked cars can also be used as computation nodes in urban computing [3]. Parked cars are usually regarded as natural roadside nodes, because they are large number, long-time staying, wide distribution, and specific location [4]. However, there are another two features of parked cars that they are energy-constrained and can leave at any time, which have been neglected by most studies before.

When a car serves as a relay node, it may leave at any time during a period of communication. Once a car leaves, the communication link in which it serves as a relay node becomes disconnected immediately. For point-to-point communication, the use of multi-relay cooperation can reduce the impact of car's departure behavior on communication quality. When a car serves as a computing node, its departure will make computing sub-task interrupted, leading to the failure of the whole computing task. Therefore, the impact of car's leaving behavior cannot be ignored.

In this study, we focus on the impact of cars' departure behavior. We choose cars in a parking lot as study objects. Because parking lots are generally located in the business district or residential area, where the communication needs are higher than remote areas. In addition, the vehicle density in parking lots is maintained at a high level for a long time. We can form clusters with vehicles close to each other, which can provide services for moving cars and pedestrians nearby. We combine the probability distribution of parking duration in [12] with the probability distribution of car's arriving time in [13] to describe car's leaving behavior, whose impacts are introduced into the derivation of outage probability and channel capacity.

The rest of this paper is organized as follows: Section II reviews related work. Section III describes the system model. The derivations of outage probability expression and link capacity expression are presented in Section IV. Section V shows the numerical results and discusses the impacts of 
leaving behavior on outage probability. Finally Section VI concludes this study and proposes future work.

\section{RELATED WORK}

At present, studies of parked cars mainly focus on relay forwarding and content caching. The idea of introducing parked cars into vehicular network has been proposed in [4], the authors found that even if a fraction of assistant parked cars could improve network connectivity significantly. The work in [5] made use of parked cars at key interaction points to overcome the signal degradation caused by buildings in the line of sight of two cars. Eckhoff et al. [5] introduced a novel scheme to emit cooperative awareness messages at low vehicle densities periodically, and used the Manhattan grid model to simulate the street environment as well as gave the obstacle model. In [10], Reis et al. found parked cars could indeed serve both as RSUs and an extension to vehicular infrastructure deployments. Besides, the work investigated how to select a suitable parked car to be an RSU, and proposed an on-line algorithm to maximize the coverage by activating the least parked cars. Su et al. [14] proposed a novel algorithm which utilized parked cars to cache content based on access pattern of content. The authors of [15] combined RSUs with parked cars to cache content, used Stackelberg game model to decide what content would be obtained from RSUs or parked cars, which minimized the expense of content delivery.

Studies above all didn't take the impact of car's departure into account. In [12], Reis et al. provided statistic analysis about survey data from the three metropolitan areas and extracted the parking behavior of individuals vehicles. The author modeled the time that a car would spend on parking as a dual Gamma stochastic process and gave the probability distribution function of it. According to real car arrival/departure data of a representative parking lot (PL), Guner et al. in [13], simulated the car's arriving times by using Weibull distribution function. In this study, we exploit the models proposed in [12], [13] to obtain car's departure pattern, which will be applied to the overall system to analyze the impact of departure behavior on system performance.

\section{SySTEM MODEL}

This study focuses on communications between mobile terminals nearby a parking lot and a base station. The mobile terminals include pedestrians and slow-moving vehicles because of heavy traffic, which always have needs of longtime communication. Assuming every car in PLs is equipped with one antenna, those cars form clusters according to the distribution of their physical location. Each cluster has one cluster head $(\mathrm{CH})$, which stores arriving time and the time its cluster members have parked. Parked vehicles themselves have no demand for communication, only forward data. Therefore, parked cars in a cluster only send and receive a small amount of control information among each other, for keeping the channels synchronized and transmitting channel state information (CSI). We assume that all cooperative parked cars forward identical data to obtain space diversity gain.

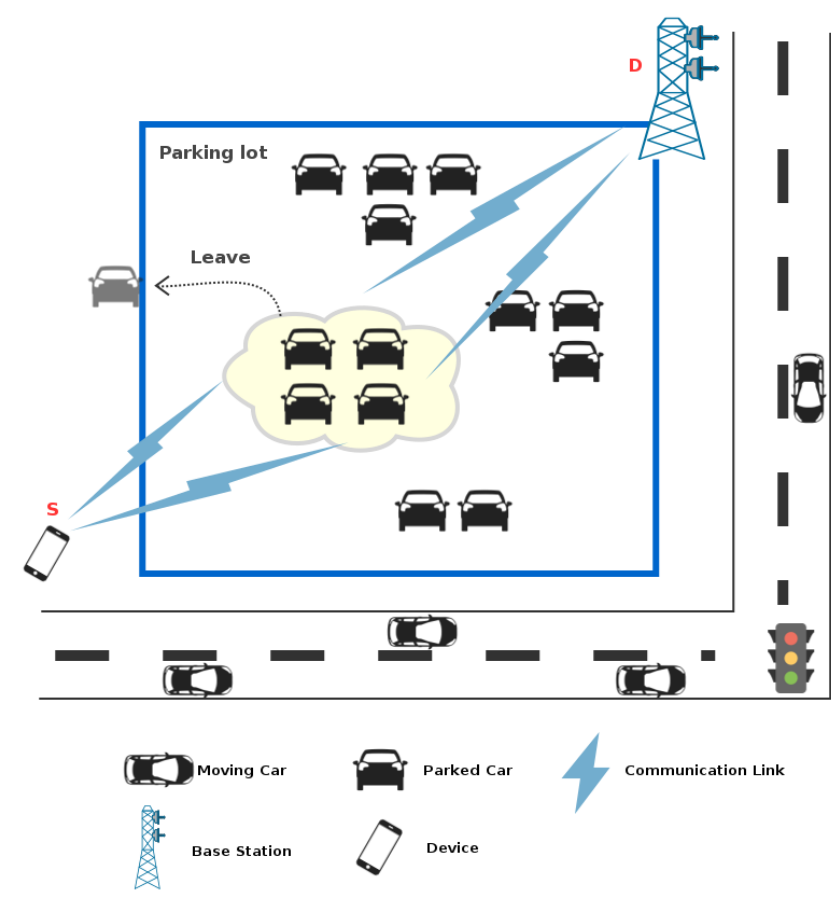

Figure 1. System Model

Consider the cooperative relays system illustrated as Figure 1 Assuming a device needs to communicate with the nearby base station, the device is the transmitter, namely $S$ in Figure 11. base station $D$ is the receiver. There is no direct link between $S$ and $D$. The transmitter selects one cluster according to information stored by $\mathrm{CHs}$ and chooses $K(K \geq 1)$ parked cars from the selected cluster. We consider half-duplex mode of operation. Relay forwarding has two phases. In phase one, the transmitter $S$ broadcasts the signal $x$ with the transmitting power $P_{\mathrm{s}}$, the receive signal at $i$-th relay is given by

$$
y_{\mathrm{sri}}=\sqrt{P_{\mathrm{s}}} h_{\mathrm{sri}} x+n_{\mathrm{sri}} i \in[1,2, \ldots, K] .
$$

where $h_{\text {sri }}$ is the channel coefficient from $S$ to the $i$-th relay and $n_{\text {sri }}$ denotes the additive white Gaussian noise (AWGN) at the $i$-th relay.

In phase two, $K$ relays amplify the identical signal and forward it to the receiver $D$, the receiving signal at $D$ from the $i$-th relay is given by

$$
y_{\text {rid }}=h_{\text {rid }} \beta_{i} y_{\text {sri }}+n_{\text {rid }} i \in[1,2, \ldots, K],
$$

where $h_{\text {rid }}$ is the channel coefficient from the $i$-th relay to $D$ and $n_{\text {rid }}$ denotes the AWGN at $D$. The relay channels are represented by $h_{\text {sri }}$ and $h_{\text {rid }}$, which are circularly symmetric complex Gaussian random variables with unit variance and zero mean. And $n_{\text {sri }}, n_{\text {rid }} \sim \mathcal{C N}\left(0, N_{0}\right)$. $\beta_{i}$ is an amplification factor that ensures the transmitting power at $i$-th relay falls below a specific value $P_{\text {ri }}$, which is given by

$$
\beta_{i}=\sqrt{\frac{P_{\mathrm{ri}}}{P_{\mathrm{s}}\left|h_{\mathrm{sri}}\right|^{2}+N_{0}}} .
$$


For single relay link, the signal-to-noise ratio (SNR) at the $i$-th relay can be represented by [16]

$$
\gamma_{i}=f\left(\frac{P_{\mathrm{s}}\left|h_{\mathrm{sri}}\right|^{2}}{N_{0}}, \frac{P_{\mathrm{ri}}\left|h_{\text {rid }}\right|^{2}}{N_{0}}\right),
$$

where $f(x, y)=\frac{x y}{1+x+y}$, and $\left|h_{\text {sri }}\right|^{2},\left|h_{\text {rid }}\right|^{2} \sim E\left(\frac{1}{2}\right)$.

The arrival time $t$ of vehicles can be described by a Weibull distribution [13], which is given by

$$
f(t)=\frac{\alpha}{\beta}\left(\frac{t}{\beta}\right)^{\alpha-1} e^{-\left(\frac{t}{\beta}\right)^{\alpha}},
$$

where $\alpha$ and $\beta$ denote shape and scale parameters. In [13], $\alpha=0.9831$ and $\beta=16.8$. The time $x$ a car will spend on parking follows a dual Gamma distribution [12], when it arrives at hour $t$. The probability density function (PDF) is given by

$$
\begin{aligned}
f(x, t)= & \frac{D_{1, t}}{\Gamma\left(\kappa_{s, t}\right) \theta_{s, t}^{\kappa_{s, t}}} x^{\kappa_{s, t}-1} e^{-\frac{x}{\theta_{s, t}}} \\
+ & \frac{D_{2, t}}{\Gamma\left(\kappa_{l, t}\right) \theta_{l, t}^{\kappa_{l, t}}} x^{\kappa_{l, t}-1} e^{-\frac{x}{\theta_{l, t}}}, \\
& x>0, t=\{0,1,2, \ldots, 23\},
\end{aligned}
$$

where $\left\{\kappa_{s, t}, \theta_{s, t}, \kappa_{l, t}, \theta_{l, t}, D_{1, t}, D_{2, t}\right\}$ are specific to car's arrival time $t$, and their values can be seen in the appendix of [12]. $\Gamma(\cdot)$ is the Gamma function.

Different from traditional static relays, parked cars may leave at any time. Therefore, for a single relay link, there are two cases where an interrupt will occur: (1) the SNR of the $i$-th link is below the threshold, whose probability is expressed as $P_{i}^{\gamma}$; (2) the $i$-th relay leaves in the communication process, whose probability is expressed as $P_{i}^{\mathrm{L}}$.

\section{Analysis of Outage Probability and Channel CAPACITY}

In this section, we analyze the impact of leaving behavior on outage and channel capacity. First, we give the expression of $P_{i}^{\mathrm{L}}$. Then, we derive the analytical expressions of outage and channel capacity on the basis of the above analysis.

\section{A. Outage Probability of The System}

In a period of communication time, relay's departure will cause the link to be interrupted. According to (6), the probability that a vehicle still has at least $n$ hours left for parking[12], when it has been parked for $t_{\mathrm{a}}$ time, is derived as (7), where $\Gamma(\cdot)$ and $\gamma(\cdot)$ are the upper and lower incomplete Gamma functions respectively. Then the probability of a car has at most $n$ hours left can be expressed by

$$
P\left[X_{\mathrm{t}} \leq t_{\mathrm{a}}+n \mid X_{\mathrm{t}}>t_{\mathrm{a}}\right]=1-P\left[X_{\mathrm{t}}>t_{\mathrm{a}}+n \mid X_{\mathrm{t}}>t_{\mathrm{a}}\right] .
$$

It can be seen in (8) that $n=0$ indicates the car leaves at the moment, the probability of which is always zero. But cars are possible to leave at any time. We try two ways to solve this contradiction:

1) Use the probability of leaving within a short time $\tau$ to represent the leaving probability at the moment. Then the probability that relay $i$ leaves at the moment can be expressed by

$$
P_{i}^{\mathrm{L}}=1-P\left[X_{\mathrm{t}}>t_{\mathrm{dur}}+\tau \mid X_{\mathrm{t}}>t_{\mathrm{dur}}\right] .
$$

2) Now that we have the PDFs of the time that a car arrives at a PL and a car's parked duration, we can set each car's arrival time and parked duration according to (5), (6). Then we can use Monte Carlo simulation to analyze the impact of departure behavior on outage probability.

For a single relay link, the cumulative distribution function (CDF) of its SNR is given in [17]

$$
\begin{aligned}
F_{\gamma_{i}}\left(x \mid w_{\text {sri }}, w_{\text {rid }}\right) & =1-2 x \sqrt{w_{\text {sri }} w_{\text {rid }}} e^{-x\left(w_{\text {sri }}+w_{\text {rid }}\right)} \\
& \times K_{1}\left(2 x \sqrt{w_{\text {sri }} w_{\text {rid }}}\right)
\end{aligned}
$$

where $K_{1}(\cdot)$ is the first order modified Bessel function of the second kind, $w_{\text {sri }}$ and $w_{\text {rid }}$ are the reciprocals of the average SNRs of the two links respectively, one is from $S$ to the $i$-th relay and the other is from the $i$-th relay to $D$. Then $w_{\text {sri }}=\frac{N_{0}}{2 P_{\mathrm{s}}}, w_{\text {rid }}=\frac{N_{0}}{2 P_{\mathrm{ri}}}$. Because the $K_{1}(x)$ function converges to $1 / x$ when $x$ approaches zero, the formula (10) can be simplified to

$$
F_{\gamma_{i}}\left(x \mid \mu_{i}\right)=1-e^{-\mu_{i} x},
$$

where $\mu_{i}=w_{\text {sri }}+w_{\text {rid }}$. The probability that the SNR of the $i$-th link is below the threshold $\gamma_{\text {th }}$ can be expressed as

$$
P_{i}^{\gamma}=F_{\gamma_{i}}\left(\gamma_{\mathrm{th}} \mid \mu_{i}\right) \text {. }
$$

On the basis of above analysis, the outage probability of the $i$-th relay link is given by

$$
\begin{aligned}
P_{i}^{\text {out }} & =f\left(P_{i}^{\gamma}, P_{i}^{\mathrm{L}}\right) \\
& =1-\left(1-P_{i}^{\gamma}\right)\left(1-P_{i}^{\mathrm{L}}\right) .
\end{aligned}
$$

To simplify the analysis, we choose select combination (SC) at the receiver. Thus, the outage probability of each link is independent of each other. And the outage probability of the two-hop communication link can be expressed as

$$
P_{\mathrm{out}}=\prod_{i \in \Omega} P_{i}^{\mathrm{out}}
$$

where $\Omega$ is the cooperative relay set.

\section{B. Capacity of The System}

We use select combination technology at the receiver for simplicity, and the SNR at the receiver is given by

$$
\gamma_{\mathrm{sc}}=\max _{i \in \Omega} \gamma_{i}
$$

where $\Omega$ is the cooperative relay set and $\gamma_{i}$ is the SNR of the $i$-th relay link. When the number of relays is $K$, the CDF of $\gamma_{\text {sc }}$ can be expressed as

$$
\begin{aligned}
F_{\gamma_{\mathrm{sc}}}(\gamma) & =P_{r}\left\{\gamma_{1}<\gamma, \gamma_{2}<\gamma, \ldots, \gamma_{\mathrm{K}}<\gamma\right\} \\
& =\prod_{i=1}^{K} F_{\gamma_{i}}\left(\gamma \mid w_{\mathrm{sri}}, w_{\mathrm{rid}}\right) .
\end{aligned}
$$




$$
P\left[X_{\mathrm{t}}>t_{\mathrm{a}}+n \mid X_{\mathrm{t}}>t_{\mathrm{a}}\right]=\frac{D_{1, t} \gamma\left(\kappa_{s, t} \frac{t_{\mathrm{a}}+n}{\theta_{s, t}}\right) \Gamma\left(\kappa_{l, t}\right)+D_{2, t} \gamma\left(\kappa_{l, t} \frac{t_{\mathrm{a}}+n}{\theta_{l, t}}\right) \Gamma\left(\kappa_{s, t}\right)-\Gamma\left(\kappa_{l, t}\right) \Gamma\left(\kappa_{s, t}\right)}{D_{1, t} \gamma\left(\kappa_{s, t}, \frac{t_{\mathrm{a}}}{\theta_{s, t}}\right) \Gamma\left(\kappa_{l, t}\right)+D_{2, t} \gamma\left(\kappa_{l, t}, \frac{t_{\mathrm{a}}}{\theta_{l, t}}\right) \Gamma\left(\kappa_{s, t}\right)-\Gamma\left(\kappa_{l, t}\right) \Gamma\left(\kappa_{s, t}\right)}
$$

Then the PDF can be obtained by deriving from the formula (16). Put (8) into (16) and we have

$$
\begin{aligned}
f_{\gamma_{\mathrm{sc}}}(\gamma) & =\sum_{i=1}^{K}\left\{f_{\gamma_{i}}(\gamma) \prod_{j=1, j \neq i}^{K} F_{\gamma_{i}}\left(\gamma \mid w_{\mathrm{sri}}, w_{\mathrm{rid}}\right)\right\} \\
& =\sum_{i=1}^{K}\left\{\mu_{i} e^{-\mu_{i} \gamma} \prod_{j=1, j \neq i}^{K}\left(1-e^{-\mu_{i} \gamma}\right)\right\} .
\end{aligned}
$$

according to $(8), \mu_{i}=w_{\text {sri }}+w_{\text {rid }}=\frac{N_{0}}{2 P_{\mathrm{s}}}+\frac{N_{0}}{2 P_{\mathrm{ri}}}$. We set $P_{\mathrm{ri}}=$ $P_{\mathrm{s}}$ for all relays, then $\mu_{i}=\bar{\mu}=\frac{N_{0}}{P_{\mathbb{S}}}$, for all $i \in\{1,2, \ldots, K\}$, and we can simplify the formula (17) to

$$
f_{\gamma_{\mathrm{sc}}}(\gamma)=K \bar{\mu} e^{-\bar{\mu} \gamma}\left(1-e^{-\bar{\mu} \gamma}\right)^{K-1} .
$$

When the transmitting power is constant, the channel capacity with $\mathrm{SC}$ can be expressed by

$$
\begin{aligned}
C & =\frac{1}{2} B \int_{\gamma}^{\infty} \log _{2}(1+\gamma) f_{\gamma_{\mathrm{sc}}}(\gamma) d \gamma \\
& =\frac{B K}{2 \ln 2} \sum_{i=1}^{K}(-1)^{i}\left(\begin{array}{c}
K-1 \\
i
\end{array}\right) e^{(i+1) \bar{\mu}} \frac{E_{1}((i+1) \bar{\mu})}{i+1}
\end{aligned}
$$

according to [18]. The coefficient $1 / 2$ is caused by two time slots occupied by one transmission, and $E_{1}(x)=$ $\int_{1}^{\infty} t^{-1} e^{-t x} d t, x>0,\left(\begin{array}{c}K-1 \\ i\end{array}\right)=\frac{(K-1) !}{(K-1-i) ! i !}$.

Due to car's leaving behavior, the number of relays will be changing during the communication. We assume that new relays are not allowed to be added during a period of communication. After a short time $\tau$, the number of relays is $K^{\prime}$, and we set $p_{i}$ as the probability that the $i$-th relay will not leave within a short time $\tau$, which equals to $1-P_{i}^{\mathrm{L}}$. Because those cars' departure events are independent to each other, we can obtain the probability distribution of $K^{\prime}$

$$
\begin{aligned}
P\left\{K^{\prime}=1\right\} & =\sum_{i=1}^{K} p_{i} \prod_{n=1, n \neq i}^{K}\left(1-p_{n}\right) \\
P\left\{K^{\prime}=2\right\} & =\sum_{i=1}^{K} \sum_{j=2}^{K} p_{i} p_{j} \prod_{n=1, n \neq i, j}^{K}\left(1-p_{n}\right) \\
& \vdots \\
P\left\{K^{\prime}=k\right\} & =\sum_{i_{1}=1 i_{2}=i_{1}+1}^{K} \sum_{i_{k}=i_{k-1}+1}^{K} p_{i_{1}} p_{i_{2}} \cdots p_{i_{k}} \\
& \times \prod_{n=1}^{K}\left(1-p_{n}\right) . \\
& n \neq i_{1}, i_{2}, \cdots, i_{k}
\end{aligned}
$$

Taking the car's leaving into consideration, the channel capacity $C_{l}$ after time $\tau$ can be written as

$$
C_{l}=\sum_{k=1}^{K} P\left\{K^{\prime}=k\right\} C .
$$

Substitute (19) and (20) into (21), we obtain the complete expression of the channel capacity as (22).

\section{Numerical Result}

This section shows the numerical evaluation of the results presented in the previous sections, followed by discussions and conclusions. The typical values of system parameters are selected from certain practical scenarios. Especially, the time $t_{\text {dur }}$ that a car has been parked for can be calculated by the subtraction of the arrival time $t_{\text {arr }}$ and current time $t_{\text {cur }}$, we set $t_{\text {cur }}=3: 00$ P.M and $t_{\text {arr }}$ is the expected value of the arrival time. The transmitting power of the sender and relays is identical $P_{\mathrm{s}}=P_{\mathrm{ri}}=2 \mathrm{~W}$.

\section{A. Outage Probability}

Figure 2 shows the outage probability trend versus SNR threshold. To verify the derivation of the outage probability, we have also conducted a simulation. The result of the simulation is presented in Figure 2 with scattering points. It is clear from the figure that for all practical purposes the two curves are essentially indistinguishable and the figure also shows the improvement in outage probability as the number of the relays increases. With multi-relays, the impact of car's departure behavior can be reduced. And the using of multirelays can reduce the transmit power of single relay in the same communication quality comparing to the using of single relay. Because cars in PLs belong to private owners, the energy consumption of each car should be as little as possible.

On the basis of PDFs of arriving time in (5) and the time a car will spend on parking in (6), Figure 3 presents the arrival/departure times for a sample day. It is seen that a large number of vehicles arrive at a PL in the morning and leave at sunset. We guess there may be a certain relationship between the system's outage probability and daily change of PLs. We assume a PL has a total parking capacity of 500 cars, which serves 2,000 cars within a day, and set the arrival time and predicted parked duration of each car by formulas, (5) and (6). Then we can obtain the change of outage probability in a day, which is shown in Figure 4. As can be seen from the curves, the outage probability decreases first and then increases. Combining with Figure 3, there are lots of cars arriving at the PL but few cars leaving from 8:00 AM to 11:00 AM. The communication link established during this period can keep active for a long time, because these cars have a long-term stay trend. Besides, the number of optional 


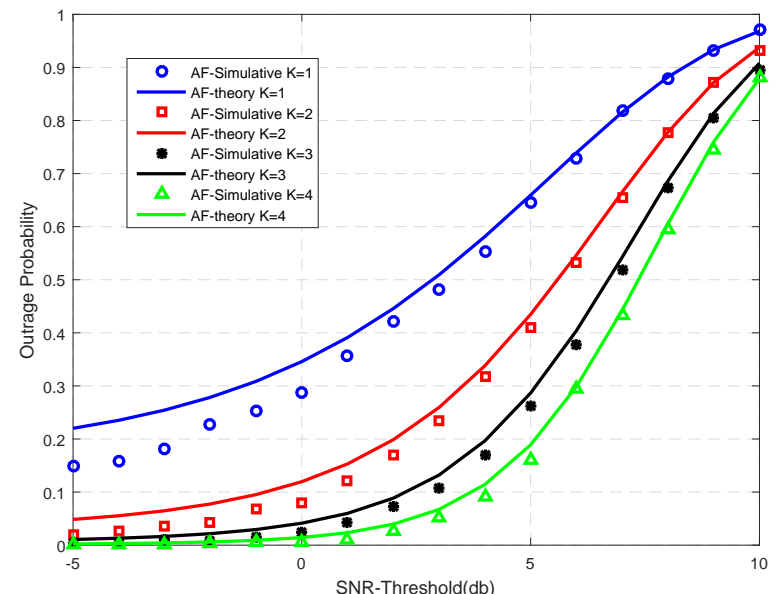

Figure 2. Outage probability versus threshold SNR for a system

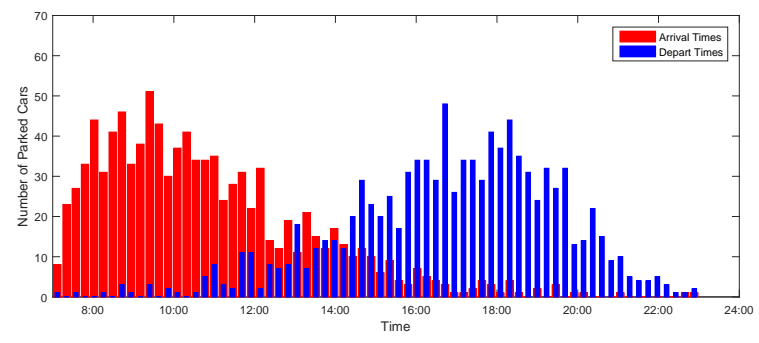

Figure 3. Arrival/departure times of the cars for a weekday

relays has increased. Thus, the outage probability decreases. Otherwise, there are lots of cars leaving from the PL but few cars arriving after 5:00 PM. During this period, the outage is easy to happen because many cars are likely to leave in the short term.

\section{B. Impact of Departure Pattern Parameters}

In this study, the departure pattern we established has two key parameters, the arrival time $t_{\text {arr }}$ and the time $t_{\text {dur }}$ a car has parked. The two parameters directly determine the probability that a car leaves at some time, thus affect the outage probability. In order to find out the impact of $t_{\text {arr }}$ and $t_{\text {dur }}$, we calculate the outage probability with only one relay forwarding data. First, we set a fixed $t_{\mathrm{arr}}$, and calculate $P_{\text {out }}$ with different $t_{\mathrm{dur}}$. Figure 5 shows the impact of $t_{\mathrm{dur}}$ on the outage probability. It can be seen that the curves almost parallel to the horizontal axis, which indicates that $t_{\mathrm{dur}}$ has little impact on $P_{\text {out }}$, even can be ignored.

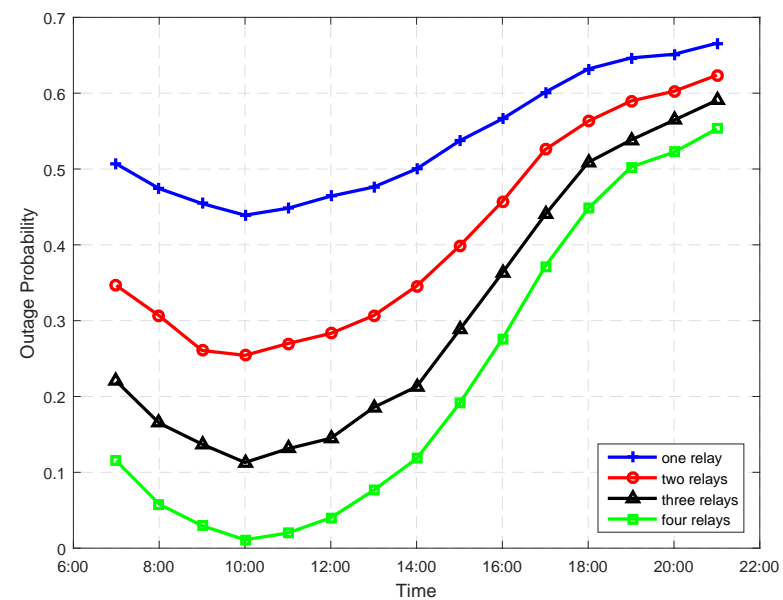

Figure 4. Outage probability in a day

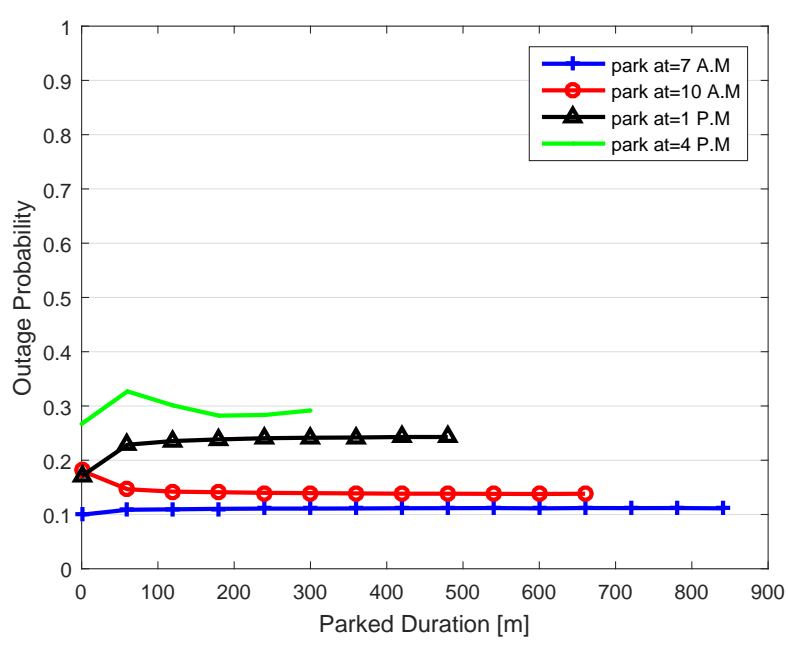

Figure 5. The outage probability versus $t_{\mathrm{dur}}$, in minutes

Then we set $t_{\text {dur }}$ fixed, the impact of $t_{\text {arr }}$ on the outage probability is shown in Figure 6. We can find that selecting cars that arrive at PLs from 4:00 P.M to 6:00 P.M as relays will lead to higher outage probability, because these cars are likely to leave in short time, while cars that arrive at earlier time are long-staying in PLs. Therefore, relay selection algorithms based on car's leaving behavior, which will be presented in the future work, should mainly focus on the impact of $t_{\mathrm{arr}}$, not the impact of $t_{\mathrm{dur}}$. 


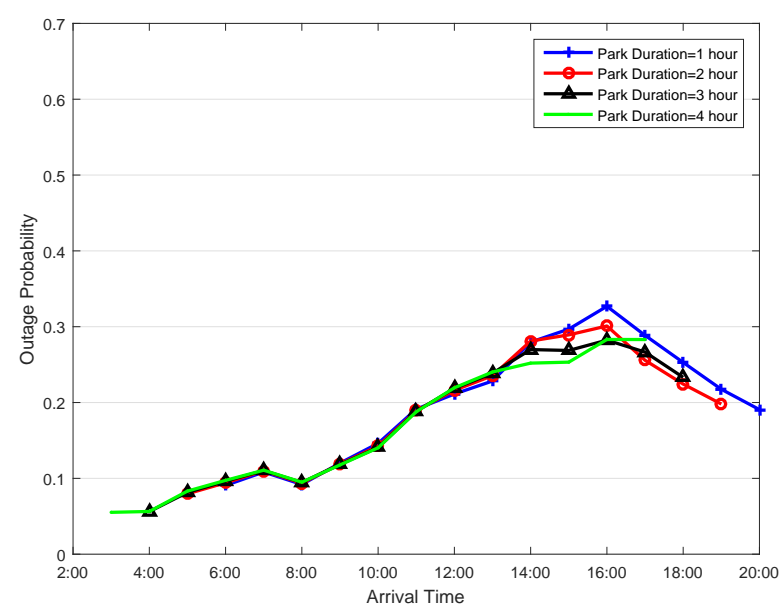

Figure 6. The outage probability versus $t_{\text {arr }}$

\section{Capacity}

To find out the change trend of the channel capacity within a day, we assume there is a PL same as the one mentioned in subsection $\mathrm{A}$, and select one relay randomly every hour, then we can obtain the change of the channel capacity in a day. Figure 7 shows the variation of the channel capacity from 7:00 A.M to 22:00 A.M. We find out that the channel capacity increases first and then decreases, which is opposite to the change trend of outage probability.

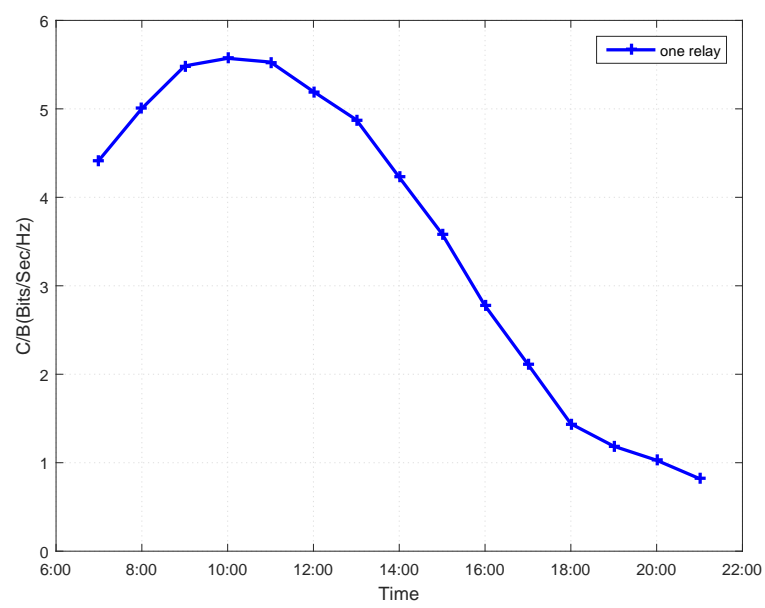

Figure 7. The channel capacity within a day

\section{Conclusions ANd Future Work}

This study considers a two-hop communication assisted by parked cars in a PL. We give an appropriate expression of car's leaving probability based on existing parking patterns. Taking SNR and cars' departure behavior into account, we derive the expressions of outage probability and channel capacity of this system. With the analysis of the change of outage probability within a day, we find that outage probability of this system is related to the traffic volume in PLs. There are two peak points of traffic volume at around 8:00 A.M and 19:00 A.M. From 8:00 A.M to 10:00 A.M, with lots of cars pouring in PLs, the number of optional cars as relays is increasing. Thus, the outage probability is low. After 4:00 P.M, with lots of cars leaving from PLs, the outage probability is increasing. We also investigate the effects caused by the parameters of the parking model, and find that the time a car has been parked for has little impact on the outage probability of this system while the impact of car's arrival time is greater.

In our system model, we assume the cluster has been formed, and relay selection is random. In the future, we focus on how to form a cluster in PLs efficiently and may propose an appropriate relay selection algorithm based on car's leaving patterns.

\section{REFERENCES}

[1] T. Han, X. Ge, L. Wang, K. S. Kwak, Y. Han, and X. Liu. 5G Converged Cell-Less Communications in Smart Cities. IEEE Communications Magazine, 55(3):44-50, March 2017.

[2] L. Wang, T. Han, Q. Li, J. Yan, X. Liu, and D. Deng. Cell-less Communications in 5g Vehicular Networks Based on Vehicle-Installed Access Points. IEEE Wireless Communications, vol. 24, no. 6, pp. 64âĂŞ71, December 2017.

[3] X. Hou, Y. Li, M. Chen, D. Wu, D. Jin, and S. Chen. Vehicular Fog Computing: A Viewpoint of Vehicles as the Infrastructures. IEEE Transactions on Vehicular Technology, 65(6):3860-3873, June 2016.

[4] N. Liu, M. Liu, W. Lou, G. Chen, and J. Cao. PVA in VANETs: Stopped cars are not silent. In 2011 Proceedings IEEE INFOCOM, pages 431435, April 2011.

[5] D. Eckhoff, C. Sommer, R. German, and F. Dressler. Cooperative Awareness at Low Vehicle Densities: How Parked Cars Can Help See through Buildings. In 2011 IEEE Global Telecommunications Conference - GLOBECOM 2011, pages 1-6, December 2011.

[6] F. Malandrino, C. Casetti, C. F. Chiasserini, C. Sommer, and F. Dressler. The Role of Parked Cars in Content Downloading for Vehicular Networks. IEEE Transactions on Vehicular Technology, 63(9):4606-4617, November 2014.

[7] J. Zhao and G. Cao. VADD: Vehicle-Assisted Data Delivery in Vehicular Ad Hoc Networks. In Proceedings IEEE INFOCOM 2006. 25TH IEEE International Conference on Computer Communications, pages 1-12, April 2006.

[8] N. Liu, M. Liu, G. Chen, and J. Cao. The sharing at roadside: Vehicular content distribution using parked vehicles. In 2012 Proceedings IEEE INFOCOM, pages 2641-2645, March 2012.

[9] W. Viriyasitavat, O. K. Tonguz, and F. Bai. UV-CAST: an urban vehicular broadcast protocol. IEEE Communications Magazine, 49(11):116124, November 2011.

[10] A. B. Reis, S. Sargento, and O. K. Tonguz. Parked Cars are Excellent Roadside Units. IEEE Transactions on Intelligent Transportation Systems, PP(99):1-13, 2017.

[11] O. K. Tonguz and W. Viriyasitavat. Cars as roadside units: self-organizing network solution. IEEE Communications Magazine, 51(12):112-120, December 2013.

[12] A. B. Reis and S. Sargento. Statistics of parked cars for urban vehicular networks. In 2016 IEEE 17th International Symposium on A World of Wireless, Mobile and Multimedia Networks (WoWMoM), pages 1-6, June 2016.

[13] S. Guner, A. Ozdemir, and G. Serbes. Impact of car arrival/departure patterns on EV parking lot energy storage capacity. In 2016 International Conference on Probabilistic Methods Applied to Power Systems (PMAPS), pages 1-5, October 2016.

[14] Z. Su, P. Ren, and X. Gan. A novel algorithm to cache vehicular content with parked vehicles applications. In 2014 IEEE International Conference on Communications (ICC), pages 5665-5669, June 2014.

[15] Z. Su, Q. Xu, Y. Hui, M. Wen, and S. Guo. A Game Theoretic Approach to Parked Vehicle Assisted Content Delivery in Vehicular Ad Hoc Networks. IEEE Transactions on Vehicular Technology, PP(99):11,2016 
[16] V. Emamian, P. Anghel, and M. Kaveh. Multi-user spatial diversity in a shadow-fading environment. In Proceedings IEEE 56th Vehicular Technology Conference, volume 1, pages 573-576 vol.1, 2002.

[17] M. O. Hasna and M. S. Alouini. End-to-end performance of transmission systems with relays over Rayleigh-fading channels. IEEE Transactions on Wireless Communications, 2(6):1126-1131, November 2003.

[18] M. S. Alouini and A. J. Goldsmith. Capacity of Rayleigh fading channels under different adaptive transmission and diversity-combining techniques. IEEE Transactions on Vehicular Technology, 48(4):11651181, July 1999

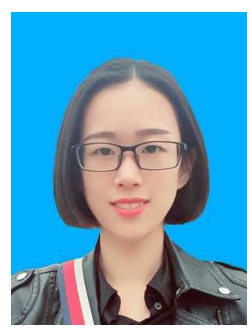

Yingying Sun received the Bachelor's degree in electronic and information engineering from Wuhan University of Technology, Wuhan, China, in 2016. She is currently working toward the Master's degree in Huazhong University of Science and Technology (HUST), Wuhan, China. Her research interests include cooperative communication and vehicular network.

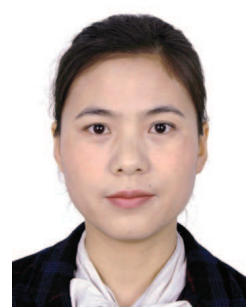

Lijun Wang received the B.S. degree in telecommunication engineering from Xidian University, Xi'an, China in July, 2004 and the M.S. degree in communication and information system from Huazhong University of Science and Technology (HUST), Wuhan, China in June, 2008. From Sept., 2016 she has been working toward the Ph.D. degree with Wuhan University, Wuhan, China.

She is currently an associate professor with the Department of Information Science and Technology, Wenhua College, Wuhan, China. Her research interests include wireless communications, and multimedia communications.

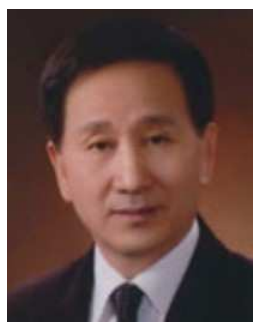

Kyung Sup Kwak received his BS degree from the Inha University, Inchon, Korea, in 1977 and his MS degree from the University of Southern California in 1981 and his $\mathrm{PhD}$ degree from the University of California at San Diego in 1988, under the Inha University Fellowship and the Korea Electric Association Abroad Scholarship Grants, respectively.

Since then, he has been with the School of Information and Communication Engineering, Inha University, Incheon, South Korea, as a Professor, and served as the Dean of the Graduate School of Information Technology and Telecommunications and the Director of the UWB Wireless Communications Research Center, an IT research center, South Korea, since 2003. In 2006, he was the President of the Korean Institute of Communication Sciences (KICS), and in 2009, the President of the Korea Institute of Intelligent Transport Systems. He published more than 200 peerreviewed journal papers and served as TPC and Track Chairs/Organizing Chairs for several IEEE related conferences. His research interests include multiple access communication systems, mobile and UWB radio systems, future IoT, and wireless body area networks: nano networks and molecular communications. In 1993, he received the Engineering College Achievement Award from Inha University, and a service award from the Institute of Electronics Engineers of Korea, in 1996 and 1999 he received distinguished service awards from the KICS. He received the LG Paper Award in 1998, and Motorola Paper Award in 2000. He received official commendations for UWB radio technology research and development from the Minister of Information and Communication, Prime Minister, and President of Korea in 2005, 2006, and 2009, respectively. In 2007, he received Haedong Paper Award and in 2009, Haedong Scientific Award of research achievement. In 2008, he was elected as an Inha Fellow Professor and currently as an Inha Hanlim Fellow Professor.

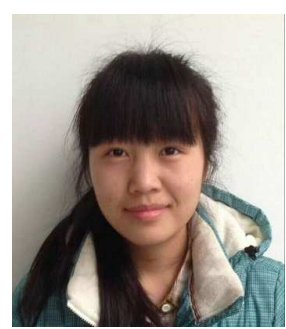

Xuming Yao received the Bachelor's degree in electronic and information engineering from Wuhan University of Technology, Wuhan, China, in 2016. She is currently working toward the Master's degree in the University of Edinburgh, Edinburgh, the United Kingdom.

Her research interests include cooperative communication and signal processing.

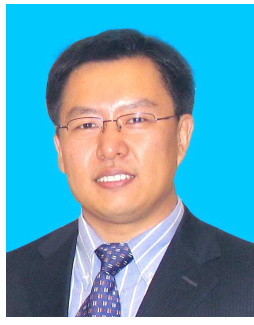

Zhiquan Bai received the M.Eng. degree in communication and information systems from Shandong University, Jinan, China, in 2003, and the Ph.D degree in communication engineering with honor from INHA University in 2007, under the Grant of Korean Government IT Scholarship, Incheon, Korea.

From 2007 to 2008, he was a postdoctor with UWB Wireless Communications Research Center, INHA University, Incheon, Korea. Since 2007, he has been an associate professor with the School of Information Science and Engineering, Shandong University, China. He has published more than 70 papers in international conferences and journals. His current research interests include cooperative communications and MIMO system, ultra wideband communications, cognitive radio system, and beyondfourth generation wireless communications.

Dr. Bai is the associate editor of the Introduction Journal of Communication Systems. He served as a TPC member and a session chair for some international conferences.

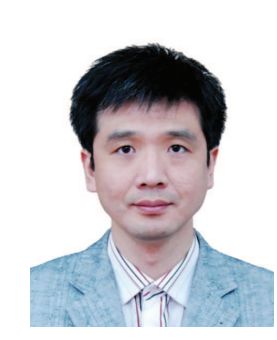

Tao Han received the Ph.D. degree in information and communication engineering from Huazhong University of Science and Technology (HUST), Wuhan, China, in 2001

$\mathrm{He}$ is currently an Associate Professor with the School of Electronic Information and Communications, HUST. From 2010 to 2011, he was a Visiting Scholar with the University of Florida, Gainesville, FL, USA, as a Courtesy Associate Professor. He has published more than 50 papers in international conferences and journals. His research interests include wireless communications, multimedia communications, and compute networks.

$\mathrm{He}$ is currently serving as an Area Editor for the European Alliance Innovation Endorsed Transactions on Cognitive Communications. 